hep-th/yymm.nnnn

November 18, 2018

\title{
More on the scalar-tensor B-F theory
}

\author{
Harvendra Singh \\ Theory Group, Saha Institute of Nuclear Physics \\ 1/AF, Bidhannagar, Kolkata 700064, India \\ E-mail: h.singh [AT] saha.ac.in
}

\begin{abstract}
This work is based on an earlier proposal [1] that the membrane B-F theory consists of matter fields alongwith Chern-Simons fields as well as the auxiliary pairs of scalar and tensor fields. We especially discuss the supersymmetry aspects of such a membrane theory. It is concluded that the theory possesses maximal supersymmetry and it is related to the L-BLG theory via a field map. We obtain fuzzy-sphere solution and corresponding tensor field configuration is given.
\end{abstract}




\section{Introduction}

Recent advances in 3-dimensional matter Chern-Simons field theories have led to some interesting proposals for the superconformal field theory describing supermembranes living in eleven spacetime dimensions. Amongst these, the BaggerLambert-Gustavsson (BLG) theory has $\mathcal{N}=8$ superconformal invariance but so far this theory has been constructed explicitly for a compact $S O(4)$ gauge group only $[2,3]$. While allowing for noncompact (Lorentzian) tri-Lie-algebras, the BLG framework has been extended further to admit full $S U(N)$ gauge symmetry [4, 5]. But these latter ones, also known as L-BLG theories, have ghost fields in their spectrum. Once the ghost fields are eliminated through gauging procedure the theory eventually reduces to the $S U(N)$ super Yang-mills theory [6]. On the other hand, another interesting class of matter Chern-Simons theories, known as ABJM theories [7], however are based on ordinary Lie-algebras involving bi-product gauge groups. The ABJM theory admits $\mathcal{N}=6, U(N)_{k} \times U(N)_{-k}$ superconformal symmetry, and these are conjectured to be dual to M-theory compactified on $A d S_{4} \times S^{7} / Z_{k}$ spacetime, with arbitrary level $k>2$. Only when $k=1,2$, the theory supposedly becomes a maximally supersymmetric theory. The $A d S_{4}$ geometry arises in the near horizon limit when $N$ M2-branes are placed at the singularity in an 8-dimensional orbifold space $C_{4} / Z_{k}[7]{ }^{1}$ The two theories BLG and ABJM complement each other but the theories have very distinct field theoretic structures though. Particularly in the context of L-BLG theories, it has become imperative to explore the fundamental importance of tri-algebras in a membrane theory. ${ }^{2}$ Along this direction there have been works where the maps between L-BLG and ABJM theories are explored in detail [10]. ${ }^{3}$ Particularly, our motivation in this paper shall be not to emphasize on the tri-algebra aspects, instead we simply try to work with ordinary Lie-algebra so long as it is possible.

Following various works $[12,13,5]$ on B-F (Chern-Simons) and L-BLG Lagrangians, in a recent paper [1] we showed that one can construct membrane B-F theories simply using ordinary Lie algebra. The crucial difference had been that unlike in the L-BLG construction which relies upon the introduction of pair of propagating (ghost) fields $\left(X^{+}, X^{-}\right)$, our construction instead requires introduction of pairs of scalar and tensor fields. The tensor fields are introduced through 'BdGPT' like field duality as in the Romans theory, it is discussed in the Appendix here.

\footnotetext{
${ }^{1}$ Specifically, M2-brane solutions on a 'resolved' $C_{4} / Z_{4}$ space and corresponding Chern-Simons level flow in ABJM theory have been studied in [8]. It is shown that the M2-brane solutions are smooth when branes are placed on the resolution.

${ }^{2}$ The primary motivation for studying 3 -algebras in membrane theory context arose from the work $[9]$

${ }^{3}$ Also specially see [11] for a divergent study of Jordan algebras in the BLG framework.
} 
Interestingly, these dual-pairs of scalar and tensor fields remain non-propagating in the action just like the Chern-Simons fields. In the present paper we work within the axiom that the $S U(N)$ membrane theory has fundamental propagating scalar fields along with auxiliary B-F gauge fields and auxiliary scalar-tensor fields and their superpartners. Incidentally, eight 2-rank tensor fields $C_{\mu \nu}^{I}$ appear only through their topological coupling with dual scalar fields $\eta^{I}$ as

$$
\int \eta^{I} d C_{(2)}^{I}
$$

The vev $<\eta^{I}>$ eventually gets related to the coupling constant of the 3D superYang-Mills theory. Thus the strength of the coupling constant determines the presence of tensor fields in the membrane BF theory. If the coupling vanishes in the vacuum so also the tensor fields. The presence of $C_{(2)}^{I}$ perhaps may also be motivated from the membrane boundary point of view. An open-membrane is a 2-dimensional extended object and its boundary (taking for example M2-brane ending on M5brane) is essentially an extended string-like configuration which can inherit a fundamental tensor field $C_{\mu \nu}$. Such one-dimensional extended solitonic excitations would of course live in the world-volume theory of M5-branes. This is essentially the argument also used by Basu and Harvey [9] in order to propose tri-algebras. We do know there are solitonic string solutions on M5-branes with self-dual 3-form tensor fields $\mathbf{C}_{\mu \nu \lambda}$ along its world-volume [15]. So when M2-branes end on M5-brane, by gauge symmetry argument, we should define a gauge invariant field strength $\left(\mathbf{C}_{\mu \nu \lambda}<\eta^{I}>-\partial_{[\mu} C_{\nu \lambda]}^{I}\right)$ on the M5-brane. ${ }^{4}$ While from M2-brane point of view the membrane having nontrivial boundary configuration should correspondingly include a tensor field $C_{\mu \nu}^{I}$ (non-propagating) in its world-volume theory, such as the coupling in (1). The above argument appears similar in spirit to the case when open-strings end on D $p$-branes. The string end-points are charged with gauge (Chan-Paton) fields which give rise to the topological (gauge) coupling

$$
g_{s} \int_{\partial \Sigma} \mathcal{A}_{(1)}
$$

in the open-string world-sheet theory and also give rise to a dynamical gauge theory on the $\mathrm{D} p$-brane itself.

Our goal in this paper is to extend our earlier work [1] and specially discuss the supersymmetry aspects of the B-F theory with tensor fields. We shall show that the theory has a maximal supersymmetry. We also discuss supersymmetric solutions, particularly the fuzzy-sphere solution, and obtain corresponding nontrivial tensor

\footnotetext{
${ }^{4}$ It is not clear whether a formulation of $M 5$-brane theory exists with this kind of field structure. However, an important covariant formulation of M5-brane theory with field structure $\left(\mathbf{C}_{\mu \nu \lambda}-\right.$ $\left.\partial_{[\mu} C_{\nu \lambda]}\right)$ and with an auxiliary scalar field has been studied in [14].
} 
field responsible for this solution. We also comment on the equivalence between our ordinary Lie-algebra theory and the tri-Lie-algebra based L-BLG theories.

The paper is organised as follows. In the section- 2 we review the main aspects and symmetries of the membrane B-F action. In the section-3 we provide a supersymmetric completion of this theory. We then discuss the equivalence between our work and the L-BLG frame work. The section-4 deals with the supersymmetric fuzzy $S^{2}$ solution and we discuss the hidden aspects of the shift-symmetry. The conclusions are given in section-5.

\section{Review: STBF theory}

The bosonic part of the membrane B-F action proposed in [1], or more appropriately called scalar-tensor B-F (STBF) action here, is given by

$$
\begin{aligned}
S_{S T B F}= & \int d^{3} x\left[\operatorname{Tr}\left(-\frac{1}{2}\left(D^{\mu} X^{I}-\eta^{I} B^{\mu}\right)^{2}+\frac{1}{2} \epsilon^{\mu \nu \lambda} B_{\mu} F_{\nu \lambda}-U(\eta, X)\right)\right. \\
& \left.-\frac{1}{2} \epsilon^{\mu \nu \lambda} C_{\mu \nu}^{I} \partial_{\lambda} \eta^{I}\right]
\end{aligned}
$$

where

$$
\begin{aligned}
& D_{\mu} X^{I}=\partial_{\mu} X^{I}-\left[A_{\mu}, X^{I}\right] \\
& V_{I J K}=\eta_{[I} X_{J K]}=\eta_{I} X_{J K}+\text { cyclic permutations of indices } \\
& U=\frac{1}{2.3 !}\left(V_{I J K}\right)^{2} .
\end{aligned}
$$

Here $X_{J K}=\left[X_{J}, X_{K}\right]$ is the Lie bracket. The $X^{I}$ 's $(I=1, \cdots, 8)$ are the scalars while $B_{\mu}$ and $A_{\mu}$ are the Chern-Simons gauge fields. All fields are in the adjoint of $U(N)$ except the scalars $\eta^{I}$ and the tensors $C_{\mu \nu}^{I}$ which are singlets. Note that tensor fields appear only as Lagrange multipliers.

Various equations of motion are: namely the $X^{I}$ equation

$$
\partial_{\mu}\left(D^{\mu} X^{I}-\eta^{I} B^{\mu}\right)-\left[A_{\mu},\left(D^{\mu} X^{I}-\eta^{I} B^{\mu}\right)\right]-\partial_{X^{I}} U=0,
$$

the $B_{\mu}$ equation (or the dNS-duality relation [16])

$$
\frac{1}{2 !} \epsilon^{\mu \nu \lambda} F_{\nu \lambda}=-\left(D^{\mu} X^{I}-\eta^{I} B^{\mu}\right) \eta^{I}
$$

the $C_{\mu \nu}^{I}$ equation

$$
\partial_{\lambda} \eta^{I}=0
$$

and the $\eta^{I}$ equation

$$
\operatorname{Tr}\left(\left(D^{\mu} X^{I}-\eta^{I} B^{\mu}\right) B_{\mu}-\frac{1}{2} V^{I J K} X_{J K}\right)+\frac{1}{2} \epsilon^{\mu \nu \lambda} \partial_{\mu} C_{\nu \lambda}^{I}=0 .
$$


Thus $\eta^{I}$ 's are constants in a given vacuum. The equation (8) does relate $\eta^{I}$ with its dual field $C_{\nu \lambda}^{I}$ and should be taken as the Hodge-duality (BdGPT) relation, literally in the same sense as in Romans' type IIA supergravity theory, see Appendix for details. In this way, the fields $\eta^{I}$ and $C_{\mu \nu}^{I}$ form dual-pair of fields. Note that, in this form of the action the scalar-tensors and the Chern-Simons $\left(B_{\mu}, A_{\mu}\right)$ fields are at the same footing. They all are auxiliary fields. So it will be more appropriate to call the above theory as scalar-tensor B-F or simply STBF membrane theory. There are however no free parameters in the theory.

The action has an scale invariance

$$
\begin{aligned}
& x_{\mu} \rightarrow a^{-1} x_{\mu}, \quad X^{I} \rightarrow a^{1 / 2} X^{I}, \quad\left(B_{\mu}, A_{\mu}\right) \rightarrow\left(a B_{\mu}, a A_{\mu}\right), \\
& \left(\eta^{I}, C_{\mu \nu}^{I}\right) \rightarrow\left(a^{1 / 2} \eta^{I}, a^{3 / 2} C_{\mu \nu}^{I}\right)
\end{aligned}
$$

where $a$ is an arbitrary scale parameter.

The gauge symmetry of the action is

$$
\begin{aligned}
& X^{I} \rightarrow U^{-1} X^{I} U, \quad A_{\mu} \rightarrow U^{-1} A_{\mu} U-U^{-1} \partial_{\mu} U, \\
& B_{\mu} \rightarrow U^{-1} B_{\mu} U,
\end{aligned}
$$

where $U \in U(N)$. Note that the $B_{\mu}$ field transforms as an adjoint field like $X^{I}$ but distinctly as compared to the gauge field $A_{\mu}$. The noncompact shift symmetry under which $X^{I}$ transforms as $X^{I} \rightarrow X^{I}+\eta^{I} M$, where $M$ is arbitrary [5], is not the symmetry of the action (3) because $\eta^{I}$ 's are not constant. However, it remains a symmetry in a given vacuum, that is when $\left\langle\eta^{I}\right\rangle$ become constant. In order to recover the shift symmetry in the action itself we will need to add compensating terms, as we discuss it next along with supersymmetry.

Note that, in the vacuum we shall have coupling constants $g^{I}$ which gets rotated under $S O(8)$. The identification of these couplings goes as

$$
g^{I}=<\eta^{I}(x)>, \quad g^{I} g^{I}=\left(g_{Y M}\right)^{2}
$$

where $g_{Y M}$ is the Yang-Mills coupling constant in the D2-brane gauge theory. The B-F action (3) has a new $U(1)$ invariance under

$$
C_{(2)}^{I} \rightarrow C_{(2)}^{I}+d \alpha_{(1)}^{I}
$$

where $\alpha_{(1)}^{I}$ are arbitrary 1-forms.

The dNS relation and the BdGPT relation in eq.(8) can be combined to give an identity

$$
\operatorname{Tr}\left(\frac{1}{2 !} \epsilon^{\mu \nu \lambda} F_{\nu \lambda} B_{\mu}+U\right)=\frac{1}{2} \eta^{I} \epsilon^{\mu \nu \lambda} \partial_{\mu} C_{\nu \lambda}^{I}
$$


This is an useful relation. It implies that there can always be a nontrivial tensor field in the vacuum whenever the gauge fields are nontrivial or when there is a nontrivial potential. Particularly, in an Abelian theory $U=0$, the gauge fields have to be present for tensor fields to be nontrivial. We shall give an example where tensor fields are nontrivial.

In summary, the B-F theory has actually two sets of pair of fields, the dNS adjoints $\left(B_{\mu}, A_{\mu}\right)$ and the BdGPT singlets $\left(\eta^{I}, C_{\mu \nu}^{I}\right)$. The introduction of these pairs has helped in bringing YM theory into the B-F Lagrangian form which has explicit $S O(8)$ global invariance and $U(N)$ gauge symmetry. In the work [1], it was left to determine what is the actual supersymmetry content of this scalar-tensor B-F theory as only bosonic part of the Lagrangian was presented there. Here we determine the full $\mathcal{N}=8$ supersymmetry content of the theory.

\section{Supersymmetry}

\subsection{The U(1) case}

To help the task we discuss the Abelian case first as the potential vanishes in this case. The Lagrangian for a single membrane can be obtained from the above STBF action and it is

$$
S_{U(1)}=\int d^{3} x\left(-\frac{1}{2}\left(\partial^{\mu} X^{I}-\eta^{I} B^{\mu}\right)^{2}+\frac{1}{2} \epsilon^{\mu \nu \lambda} B_{\mu} F_{\nu \lambda}-\frac{1}{2} \epsilon^{\mu \nu \lambda} C_{\mu \nu}^{I} \partial_{\lambda} \eta^{I}-\partial_{\lambda} \eta^{I}\left(B^{\lambda} X^{I}\right)\right)
$$

Note that an additional term $-\partial_{\lambda} \eta^{I}\left(B^{\lambda} X^{I}\right)$ has been added to the action (14) so that it now has a shift (Stueckelberg) symmetry

$$
\delta_{1} B_{\mu}=\partial_{\mu} f, \quad \delta_{1} X^{I}=\eta^{I} f, \quad \delta_{1} C_{\mu \nu}^{I}=\epsilon_{\mu \nu \lambda} \partial^{\lambda}\left(f X^{I}\right),
$$

in addition to the Abelian gauge invariance under the variation

$$
\delta_{2} A_{\mu}=\partial_{\mu} \lambda
$$

With the information about the supersymmetric scalar-tensor topological action given in Appendix,

$$
S_{S T}=-\int d^{3} x\left(\frac{1}{2} \epsilon^{\mu \nu \lambda} C_{\mu \nu}^{I} \partial_{\lambda} \eta^{I}+i \bar{\chi}^{A} \zeta^{A}\right),
$$

we find that a supersymmetrised Abelian STBF action is

$$
\begin{aligned}
S_{U(1)}= & \int d^{3} x\left(-\frac{1}{2}\left(\partial^{\mu} X^{I}-\eta^{I} B^{\mu}\right)^{2}+\frac{1}{2} \epsilon^{\mu \nu \lambda} B_{\mu} F_{\nu \lambda}\right. \\
& \left.-\partial_{\lambda} \eta^{I}\left(B^{\lambda} X^{I}\right)+\frac{i}{2} \bar{\psi} \not \partial \psi+i \bar{\chi} \beta \psi+S_{S T}\right)
\end{aligned}
$$


where $\psi^{A}(A=1, \cdots, 8)$ is the standard fermionic superpartner of $X^{I}$. These are 2-component Majorana spinors which also transform under $8_{s}$ spinor representation of $S O(8)$. While spinors $\chi$ and $\zeta$ make the supersymmetric partners of $\eta^{I}$ and $C_{(2)}^{I}$ respectively. Note that, in this formulation we have scalar-tensor action and the B-F (Chern-Simons) gauge actions at an equal footing. They are both topological in nature and only propagating fields are the matter fields $X^{I}$ 's.

With this action, we obtain the following $\mathcal{N}=8$ supersymmetry variations for the fields ${ }^{5}$

$$
\begin{aligned}
& \delta X^{I}=i \bar{\epsilon} \tilde{\Gamma}^{I} \psi, \quad \delta \psi=-\left(\not \partial X^{I}-\eta^{I} \not B\right) \Gamma^{I} \epsilon \\
& \delta A_{\mu}=\frac{i}{2} \eta^{I} \bar{\epsilon} \gamma_{\mu} \tilde{\Gamma}^{I} \psi-\frac{i}{2} X^{I} \bar{\epsilon} \gamma_{\mu} \tilde{\Gamma}^{I} \chi, \quad \delta B_{\mu}=0 \\
& \delta \eta^{I}=i \bar{\epsilon} \tilde{\Gamma}^{I} \chi, \quad \delta \chi=-\not \partial \eta^{I} \Gamma^{I} \epsilon, \\
& \delta C_{\mu \nu}^{I}=i \tilde{\epsilon}^{I} \gamma_{\mu \nu} \zeta, \quad \delta \zeta=\left(\not \partial\left(B X^{I}\right)+\frac{1}{2} \epsilon^{\mu \nu \lambda} \partial_{\mu} C_{\nu \lambda}^{I}\right) \Gamma^{I} \epsilon
\end{aligned}
$$

under which $U(1)$ action (18) remains invariant. The supersymmetry parameters $\epsilon^{\dot{A}}$ are eight 2-component real spinors belonging to the $8_{c}$ representation of $S O(8)$.

\subsection{The triviality of $U(1)$}

It would be useful to verify that the Abelian case presented above is nothing but the rewriting of the non-interacting theory of scalar fields describing the transverse motion of a membrane. For working this out, we first integrate out the auxiliary tensor field by using its equation of motion $\partial_{\mu} \eta^{I}=0$. So we substitute $\eta^{I}=g^{I}$ in the action. The action becomes

$$
S_{U(1)}=\int d^{3} x\left(-\frac{1}{2}\left(\partial^{\mu} X^{I}-B^{\mu} g^{I}\right)^{2}+\frac{1}{2} \epsilon^{\mu \nu \lambda} B_{\mu} F_{\nu \lambda}+\frac{i}{2} \bar{\psi} \not \partial \psi\right)
$$

Notice that now we have the Stueckelberg invariance namely:

$$
\delta X^{I}=g^{I} f(x), \quad \delta B_{\mu}=f(x) .
$$

We have two possibilities here either we integrate out $B_{\mu}$ or integrate out $A_{\mu}$ first.

I) Let us first take the case of integrating out the $A_{\mu}$ field. We presume that field strength $F_{\mu \nu}$ to be a fundamental field and impose its Bianchi identity by adding a Lagrange multiplier term $\frac{1}{2} \int \partial_{\mu} \tau F_{\nu \lambda} \epsilon^{\mu \nu \lambda}$. Here $\tau$ is periodic $\tau \sim \tau+1$. The Abelian action then becomes

$$
S_{U(1)}=\int d^{3} x\left(-\frac{1}{2}\left(\partial^{\mu} X^{I}-g^{I} B^{\mu}\right)^{2}+\frac{1}{2} \epsilon^{\mu \nu \lambda}\left(B_{\mu}+\partial_{\mu} \tau\right) F_{\nu \lambda}+\frac{i}{2} \bar{\psi} \not \partial \psi\right)
$$

\footnotetext{
${ }^{5}$ In our convention $\gamma_{\alpha \beta}^{\mu}$ are real and commute with $\Gamma_{A \dot{A}}^{I}$. The spinors $\bar{\chi}=\chi^{T} \gamma^{0}$, see Appendix for details.
} 
We then integrate out the $A_{\mu}$ which is auxiliary gauge field through the equation of motion $B_{\mu}+\partial_{\mu} \tau=0$.

$$
S_{U(1)}=\int d^{3} x\left(-\frac{1}{2}\left(\partial^{\mu} X^{I}+g^{I} \partial_{\mu} \tau\right)^{2}+\frac{i}{2} \bar{\psi} \not \partial \psi\right)
$$

Since $\tau$ transforms as $\tau \rightarrow \tau-\lambda$ under $B_{\mu} \rightarrow B_{\mu}+\partial_{\mu} \lambda$, using this freedom we can always gauge fix $\tau=0$. We are left with

$$
S_{U(1)}=\int d^{3} x\left(-\frac{1}{2}\left(\partial^{\mu} X^{I}\right)^{2}+\frac{i}{2} \bar{\psi} \not \partial \psi\right)
$$

which is nothing but the known non-interacting $S O(8)$ theory for single membrane and accounts for all the degrees of freedom. The $X^{I}$ 's are the modes describing the transverse motion of a membrane on $R^{8}$.

II) The second option could have been that we integrate out $B_{\mu}$ field first by using the dNS equation. In which case $B_{\mu}$ field eats up one of the $X^{I}$ 's through shift symmetry and it becomes heavy which also breaks $S O(8)$ spontaneously. After substituting dNS equation we obtain the gauge action representing a single D2-brane

$$
S_{U(1)}=\int d^{3} x\left(-\frac{1}{2} \sum_{i=1}^{7}\left(\partial^{\mu} X^{i}\right)^{2}-\frac{1}{4 g_{0}^{2}} F_{\mu \nu} F^{\mu \nu}+\frac{i}{2} \bar{\psi} \not \partial \psi\right)
$$

and it has explicit $S O(7)$ invariance. It is obvious that both of these actions (23) and (24) are equivalent in $3 \mathrm{D}$.

\section{$3.3 \mathrm{U}(\mathrm{N})$ case}

Now having studied the simpler Abelian case in the STBF formulation, we now set to determine the fermionic content of the non-Abelian action (3). We find that the fermionic content in the action remains the same as in Abelian case except that now $\psi^{A}$ is in the adjoint of $U(N)$, while the pair $\left(\chi^{A}, \zeta^{A}\right)$ remains gauge singlet. But there are also additional fermionic terms. The full action can be written as

$$
\begin{aligned}
S_{U(N)}=\int d^{3} x & {\left[\operatorname{Tr}\left(-\frac{1}{2}\left(D^{\mu} X^{I}-\eta^{I} B^{\mu}\right)^{2}-U(\eta, X)-\left(B^{\lambda} X^{I}\right) \partial_{\lambda} \eta^{I}+\frac{1}{2} \epsilon^{\mu \nu \lambda} B_{\mu} F_{\nu \lambda}\right)\right.} \\
& -\frac{1}{2} \epsilon^{\mu \nu \lambda} C_{\mu \nu}^{I} \partial_{\lambda} \eta^{I}+\operatorname{Tr} \frac{i}{2} \bar{\psi} \not D \psi+i \operatorname{Tr} \bar{\chi}(B \psi)-i \bar{\chi} \zeta \\
& \left.-\operatorname{Tr} \frac{i}{2} \bar{\psi} \Gamma_{I J} \eta^{I}\left[X^{J}, \psi\right]-\operatorname{Tr} \frac{i}{2} \bar{\psi} \Gamma_{I J}\left[X^{I}, X^{J}\right] \chi\right]
\end{aligned}
$$

All the spinors transform under $8_{s}$ of the R-symmetry group $S O(8)$ as usual. The covariant fermionic derivative is given by

$$
D_{\mu} \psi=\partial_{\mu} \psi-\left[A_{\mu}, \psi\right] .
$$


All bosonic covariant derivatives in the action are usual gauge covariant derivatives involving $A_{\mu}$.

Note that, the action (25) has a shift (gauge) invariance under which tensor fields also transform

$$
\begin{gathered}
\delta_{1} B_{\mu}=D_{\mu} f, \quad \delta_{1} X^{I}=\eta^{I} f, \quad \delta_{1} C_{\mu \nu}^{I}=\epsilon_{\mu \nu \lambda} \operatorname{Tr}^{\lambda}\left(f X^{I}\right), \\
\delta_{1} \psi=f \chi, \quad \delta_{1} \zeta=-\operatorname{Tr} \not \partial(f \psi) .
\end{gathered}
$$

One will easily notice that $\delta_{1} U=0$. That is the shifts $\delta_{1} X^{I}$ do not change the potential. In addition there is an usual $U(N)$ gauge symmetry involving $A_{\mu}$ fields as discussed in the review section.

Determining the supersymmetric variations for non-Abelian case is rather difficult. But we know that our theory can be mapped into L-BLG, see the section (3.4) below, so the task becomes easier. We take the lead from L-BLG work [5] and following the map in the section (3.4) we determine that the supersymmetry variations for the non-Abelian STBF are

$$
\begin{aligned}
& \delta X^{I}=i \bar{\epsilon} \tilde{\Gamma}^{I} \psi, \quad \delta \psi=-\left(\not D X^{I}-\eta^{I} \not B\right) \Gamma^{I} \epsilon-\frac{1}{3 !} V^{I J K} \Gamma_{I J K} \epsilon \\
& \delta A_{\mu}=\frac{i}{2} \eta^{I} \bar{\epsilon} \gamma_{\mu} \tilde{\Gamma}^{I} \psi-\frac{i}{2} X^{I} \bar{\epsilon} \gamma_{\mu} \tilde{\Gamma}^{I} \chi, \quad \delta B_{\mu}=i \bar{\epsilon} \gamma_{\mu} \tilde{\Gamma}_{I}\left[X^{I}, \psi\right] \\
& \delta \eta^{I}=i \bar{\epsilon} \tilde{\Gamma}^{I} \psi, \quad \delta \chi=-\not \partial \eta^{I} \Gamma^{I} \epsilon, \quad \delta C_{\mu \nu}^{I}=i \bar{\epsilon} \tilde{\Gamma}^{I} \gamma_{\mu \nu} \zeta \\
& \delta \zeta=\operatorname{Tr}\left(\not \partial\left(B X^{I}\right) \Gamma^{I}-\frac{1}{2}\left(\not \partial X^{I}\right) X^{J K} \Gamma^{I J K}\right) \epsilon+\frac{1}{2} \epsilon^{\mu \nu \lambda} \partial_{\mu} C_{\nu \lambda}^{I} \Gamma^{I} \epsilon .
\end{aligned}
$$

One can check that the straightforward reduction of (28) to the Abelian case gives the susy variations determined in the previous section. We note that at no stage did we require to invoke a tri-algebra, as all expressions in the action, including the expressions like $V^{I J K}$ or $\operatorname{Tr}\left(\not \partial X^{I}\right) X^{J K}$ in the susy variations, do involve normal Lie-brackets. ${ }^{6}$ As an important next step, we will now show that the STBF theory can actually be mapped to the familiar L-BLG theory where 3-algebra structure becomes a favorable simplifying tool.

\subsection{Generalised dNS relation, gauge fixing: Equivalence of STBF and BLG theory}

There has been an expectation that the STBF theory constructed via tensor field inclusion method must be related to L-BLG tri-algebra theory somehow. ${ }^{7}$ Particu-

\footnotetext{
${ }^{6}$ To make it clear that, although by looking at various triple products one would like to believe that these terms may come from some hidden tri-algebra structure, but it is not immediately clear if this will be true while we are in STBF set up, i.e. having tensor fields explicitly in the action. In order to realise 3-algebra explicitly we should first dualise or map STBF back to the L-BLG.

${ }^{7}$ I am grateful to Neil Lambert for raising this issue and for sharing his insight.
} 
lalrly the STBF action has got a lot of similarity with the L-BLG action [5] involving the propagating ghost fields. Here we try to establish this missing equivalence between the STBF and the L-BLG. Let us separate the bosonic STBF Lagrangian in the following manner

$$
L_{0}\left(X^{I}, \eta^{I}, B_{\mu}, A_{\mu}\right)-\frac{1}{2} \epsilon^{\mu \nu \lambda} \partial_{\mu} \eta^{I} C_{\nu \lambda}^{I}
$$

where $L_{0}$ contains all the terms in STBF Lagrangian except the tensor fields. With out any loss of the content we can introduce new set of gauge fields $\hat{A}_{\mu}^{I}$ (having mass dimension $\frac{1}{2}$ and transforming in the $8_{v}$ ) through a total derivative term

$$
L_{0}\left(X^{I}, \eta^{I}, B_{\mu}, A_{\mu}\right)-\frac{1}{2} \epsilon^{\mu \nu \lambda} \partial_{\mu} \eta^{I}\left(C_{\nu \lambda}^{I}-2 \partial_{\nu} \hat{A}_{\lambda}^{I}\right)
$$

These eight gauge fields are the singlets of $U(N)$. However, it is important to notice that these do not modify any of the equations obtained previously from STBF Lagrangian and actually these fields are just a kind of harmless spectator fields. However, due to these, action (30) has additional shift symmetry;

$$
\delta C_{(2)}^{I}=d \alpha_{(1)}^{I}, \quad \delta \hat{A}_{(1)}^{I}=\alpha_{(1)}^{I} .
$$

This invariance can be utilised to gauge fix the gauge field $\hat{A}_{\mu}^{I}=0$. This is what we have considered throughout in the paper. The BdGPT relation remains unchanged and it is

$$
\frac{\delta L_{0}}{\delta \eta^{I}}=-\frac{1}{2 !} \partial_{\mu} C_{\nu \lambda}^{I} \epsilon^{\mu \nu \lambda}
$$

To recall the dNS duality relation involving adjoint fields is

$$
\frac{1}{2 !} \epsilon^{\mu \nu \lambda} F_{\nu \lambda}=\left(\eta^{I} B^{\mu}-D^{\mu} X^{I}\right) \eta^{I}+X^{I} \partial^{\mu} \eta^{I}
$$

which defines relationship between gauge fields $A_{\mu}, B_{\mu}$ and the scalars $X^{I}$ all in adjoint of the gauge group. But it does not involve any tensor fields.

We now wish to define a 'generalised' dNS (gdNS) duality relation involving only singlet fields, namely

$$
\frac{1}{2} \epsilon^{\mu \nu \lambda}\left(C_{\nu \lambda}^{I}-\hat{F}_{\nu \lambda}\right) \equiv\left(\hat{C}^{I \mu}-\partial^{\mu} X_{-}^{I}\right)
$$

where field strength $\hat{F}^{I} \equiv d \hat{A}^{I}$. Only difference in gdNS relation and the dNS equation is that the gdNS equation involves 2-rank tensor fields along with Abelian gauge fields $\hat{C}_{\mu}^{I}, \hat{A}_{\mu}^{I}$ and the scalar $X_{-}^{I}$. The $\hat{A}_{\mu}^{I}$ need not explicitly appear in the action as it can be eaten up by the tensor field, while its presence only adds to total derivative 
terms in the action. Using this generalised duality relation, the STBF action (30) can now be written in the L-BLG form

$$
L_{0}\left(X^{I}, \eta^{I}, B_{\mu}, A_{\mu}\right)-\partial_{\mu} X_{+}^{I}\left(\hat{C}^{I \mu}-\partial^{\mu} X_{-}^{I}\right)
$$

where we redefined $\eta^{I} \equiv X_{+}^{I}$ for identification. This is the action constructed in $[5,13,6]$. Since here both $X_{+}^{I}, X_{-}^{I}$ are propagating fields with lightlike metric, due to this the L-BLG action has ghost degrees of freedom, which are eliminated through the gauge fixing as discussed in $[6,5]$. With $S U(N)$ gauge symmetry the BLG theory has been shown to acquire a Lorentzian tri-Lie-algebra structure [5].

So far that was for mapping the bosonic content on the two sides. The fermionic content is mapped as follows. Specifically, if the fields $C_{\mu \nu}^{I}, \hat{C}_{\lambda}^{I}, X_{-}^{I}$ are chosen to have their fermionic partners given by $\zeta, \hat{\chi}, \psi_{-}$respectively. Then the fermionic map from STBF to L-BLG is given by

$$
\zeta=\hat{\chi}-\not \partial \psi_{-}
$$

All other fermions remain unchanged under this map. We note that, we could make a gauge choice $\hat{A}_{\mu}^{I}=0$, likewise we can also have a choice where we can set $X_{-}^{I}=0=\psi_{-}$in L-BLG, see [6].

\section{Supersymmetric Vacua}

The moduli space of vacua in the STBF theory is larger than the 3D super YangMills theory. Our main aim is to determine vacua which will have nontrivial tensor backgrounds.

The first set of solutions are the constant $X^{I}$ configurations where $B_{\mu}$ and $A_{\mu}$ fields are vanishing $[5,1]$. So for these solutions $D_{\mu} X^{I}-\eta^{I} B_{\mu}=0$. If we take $\eta^{I}=g^{I}$ and $C_{\mu \nu}^{I}$ being constants in the vacuum, we only require

$$
X^{I J}=\left[X^{I}, X^{J}\right]=0
$$

That means $X^{I}$ 's must be commuting (diagonal) $N \times N$ matrices. It gives the moduli space to be exactly that of $N$ M2-branes on flat $R^{8}$. Since for these solutions the $V_{I J K}$ and $\left(D_{\mu} X^{I}-\eta^{I} B_{\mu}\right)$ are vanishing thus all supersymmetric fermionic variations altogether vanish. So these make the maximally supersymmetric solutions of STBF theory. These STBF vacua are the same as those of L-BLG [5] and it is consistent with the map discussed in section (3.4). We comment that for any finite coupling $\left(g^{I}\right)^{2}$ the theory actually describes the super Yang-Mills theory of D2-branes, the membrane theory is obtained only in the strong coupling limit of it as elaborated in $[5]$. 


\section{Noncommuting solutions:}

I) An interesting case arises when $C_{(2)}^{I}$ is taken to be nontrivial. For this let us take the tensor components to be dependent on the spatial coordinates

$$
d C_{(2)}^{I}=m^{I}(x) d x^{0} \wedge d x^{1} \wedge d x^{2}
$$

where $m^{I}(x)$ is a function which we shall determine next. We still take $A_{\mu}=0$ and first discuss the case with $B_{\mu}=0$. Following from $\eta^{I}$ equation of motion, we find $X^{I}$ and $m^{I}$ will be related via

$$
\frac{1}{2 !} g^{[I} \operatorname{Tr}\left(X^{J K]} X_{J K}\right)=m^{I}
$$

This ought to describe a noncommuting (fuzzy) configuration of membranes. We further simplify to the special case where $\left(\eta^{8}=g_{Y M}, \eta^{i}=0\right)$ and $\left(m^{8}=m(x), m^{i}=\right.$ $0)$. The $X^{I}$ equations of motion reduce to

$$
\begin{aligned}
& \partial_{\mu} X^{8}=0 \\
& \partial_{\mu} \partial^{\mu} X^{i}+\left(g_{Y M}\right)^{2}\left[X^{i j}, X^{j}\right]=0
\end{aligned}
$$

and following from (38), $X^{i}$ s are to satisfy the constraint

$$
\frac{1}{2} g_{Y M} \operatorname{Tr}\left(X^{i j} X_{i j}\right)=m(x)
$$

Thus $X^{8}$ has to be constant and the $X^{i}$ equations are in fact satisfied by the Nahm equation

$$
\partial_{\sigma} X^{i}=\frac{1}{2} g_{Y M} \epsilon^{i j k}\left[X^{j}, X^{k}\right] \quad(i=1,2,3)
$$

where $x_{2} \equiv \sigma$. The Nahm equation has a simple solution

$$
X^{i}=\frac{1}{g_{Y M} \sigma} \Sigma^{i}
$$

where $\Sigma^{i}$ form an $S U(2)$ subalgebra, $\left[\Sigma^{i}, \Sigma^{j}\right]=\epsilon^{i j k} \Sigma^{k}$, and are in the $N \times N$ representation. The $X^{i}(\sigma)$ are well defined for $\sigma>0$. The $\sigma=0$ is the location of the boundary brane. From eq.(40) we determine (for large N)

$$
m(\sigma) \propto \frac{N^{3}}{g_{Y M}^{3} \sigma^{4}} .
$$

It suggests that the 0-1 component of the tensor field falls off as

$$
C_{01} \propto\left(\frac{N}{g_{Y M} \sigma}\right)^{3} .
$$


Thus we have seen that the nontrivial tensor fields can be present in the STBF theory for a noncommuting fuzzy sphere configuration. It can be interpreted as an indication of the presence of the boundary M5-brane. Actually from M5-brane point of view $\sigma$ is a transverse coordinate and $\sigma \rightarrow 0$ is like probing the region where boundary branes are located.

We can express

$$
\left[X^{i}, X^{j}\right]=\frac{1}{g_{Y M} \sigma} \epsilon^{i j k} X^{k}
$$

The physical radius square of the sphere (for large $N$ ) at a fixed location $\sigma$ is

$$
r(\sigma)=\sqrt{\frac{1}{N} \operatorname{Tr}\left(\sum_{i} X_{i}^{2}\right)} \sim \frac{N}{2 g_{Y M} \sigma} .
$$

So the radius of the sphere varies with the location, $\sigma$, and it blows up near the boundary $\sigma=0$, the location of M5-brane. These fuzzy sphere solutions have earlier been obtained in D1-D3 system [17] and BMN-matrix model [18].

II) Although in the above we have taken $B_{\mu}=0, X^{8}=$ constt while solving for the fuzzy solution, instead we can take $B_{\mu}$ to be pure gauge such that it solves $\partial_{\sigma} X^{8}-g_{Y M} B_{\sigma}=0$. The fuzzy-sphere configuration above is still a solution with

$$
\frac{1}{2} g_{Y M} \operatorname{Tr}\left(X^{i j} X_{i j}\right)=\partial^{\lambda} \operatorname{Tr}\left(B_{\lambda} X^{8}\right)=\frac{1}{g_{Y M}} \operatorname{Tr} \partial_{\sigma}\left(X^{8} \partial_{\sigma} X^{8}\right)
$$

but with a constant tensor field. We then determine that

$$
X^{8} \sim \frac{1}{\sqrt{3}} \sum_{i=1}^{3} X^{i}
$$

These two fuzzy-sphere solutions, one with nontrivial tensor field and the other with a constant or vanishing value, are not related via infinitesimal (shift) symmetry as discussed in eq.(27). Hence we conclude that there can be a nontrivial tensor field background for the fuzzy sphere solution in the membrane B-F theory.

We also check that for the sphere solution all fermionic variations (28) can be made to vanish identically. Note that, from $\delta_{s} \psi=0$ the arbitrary spinors need to satisfy

$$
\gamma_{\beta \alpha}^{2} \Gamma_{\dot{B} \dot{A}}^{1238} \epsilon^{\dot{A} \alpha}=-\epsilon^{\dot{B} \beta}
$$

where $\Gamma_{\dot{B} \dot{A}}^{1238}$ is a lower diagonal component of $16 \times 16$ matrix

$$
\bar{\Gamma}_{p}=\bar{\Gamma}_{1} \bar{\Gamma}_{2} \bar{\Gamma}_{3} \bar{\Gamma}_{8}=\left(\begin{array}{cc}
\tilde{\Gamma}_{B A}^{1238} & 0 \\
0 & \Gamma_{\dot{B} \dot{A}}^{1238}
\end{array}\right)
$$


In our conventions $\Gamma_{\dot{B} \dot{A}}^{1238}=-\sigma_{2} \times \mathbf{1}_{2} \times \sigma_{2}$ and the property that $\left(\bar{\Gamma}_{p}\right)^{2}=1$. Corresponding to (49) the 32 component Weyl spinors $\hat{\epsilon}=(0, \epsilon)$ would then satisfy ${ }^{8}$

$$
\hat{\gamma}^{2} \hat{\Gamma}^{1238} \hat{\epsilon}=-\hat{\epsilon}
$$

Thus $\hat{\gamma}^{2} \hat{\Gamma}^{1238}$ will act as a projector for an arbitrary Weyl spinor $\hat{\epsilon}$. We can always choose the eigenvalues such that the 8 components of the spinors remain intact. Likewise other fermionic variations also identically vanish. Thus we find that fuzzy sphere is a $1 / 2$-supersymmetric solution of STBF theory. This is in agreement with the other known cases of fuzzy 2-sphere in the literature [17, 18].

\section{Conclusion}

We have discussed the supersymmetrisation of the membrane B-F theory (STBF) having dual-pairs of non-propagating scalar and tensor fields. The construction has been based on ordinary Lie-algebra structure. This construction leads us to a supermembrane theory with tensor fields which has $U(N)$ gauge symmetry, $S O(8)$ Rinvariance as well as the scale invariance. There are no free parameters in the action as those can be scaled away. The theory does not have propagating ghost degrees of freedom as the tensor fields are topological in nature. However, we do find that a Lorentzian tri-algebra structure can emerge if the tensor fields are dualised into propagating scalar fields via a 'generalised' dNS like duality and as a consequence the theory goes over to the known L-BLG formulation [5]. We have explicitly shown that there exists a fuzzy $S^{2}$ solution which supports a nontrivial 2-rank tensor field and it is $1 / 2$-supersymmetric. It will be interesting if we can find a fuzzy $S^{3}$ solution in STBF theory.

\section{Acknowledgements:}

I am thankful to Anirban Basu and Bobby Ezhuthachan for useful discussions. I am also grateful to Neil Lambert for the careful reading of an earlier draft and the helpful comments. Finally I wish to thank the organisors of the Indian Strings Meeting ISM'08, Pondicherry for providing an exciting environment during the workshop.

\section{A Conventions:}

The 3D Clifford algebra with signature $(-++)$ is given by

$$
\left\{\gamma^{\mu}, \gamma^{\nu}\right\}=2 \eta^{\mu \nu}
$$

\footnotetext{
${ }^{8}$ Here $\hat{\gamma}^{\mu}=\gamma^{\mu} \times \Gamma^{c h}$, with $\Gamma^{c h}=\operatorname{diag}\left(-\mathbf{1}_{8}, \mathbf{1}_{8}\right)$ being the chirality operator. While $\hat{\Gamma}^{1238}=$ $\mathbf{1}_{2} \times \bar{\Gamma}_{p}$.
} 
We choose a real representation where $\gamma^{0}=i \tau^{2}, \gamma^{1}=\tau^{1}$ and $\gamma^{2}=\tau^{3}$. The $\tau^{\prime}$ s are the Pauli matrices. The matrix $C=\gamma^{0}$ and satisfies $C \gamma^{\mu} C^{-1}=-\left(\gamma^{\mu}\right)^{T}$. The fermionic invariants can be constructed involving 2-component Majorana spinors as

$$
\bar{\chi} \zeta=\chi^{T} \gamma^{0} \zeta=\bar{\zeta} \chi, \quad \bar{\chi} \gamma^{\mu} \zeta=-\bar{\zeta} \gamma^{\mu} \chi, \quad \bar{\chi} \gamma^{\mu \nu} \zeta=-\bar{\zeta} \gamma^{\mu \nu} \chi, \cdots
$$

The $\gamma$-commutators are defined as

$$
\gamma^{\mu \nu}=\frac{1}{2}\left[\gamma^{\mu}, \gamma^{\nu}\right]=\epsilon^{\mu \nu \lambda} \gamma_{\lambda}, \quad \gamma^{\mu \nu \lambda}=\epsilon^{\mu \nu \lambda} \mathbf{1}
$$

while the Levi-Civita tensor is $\epsilon^{012}=1$.

For the internal space the $S O(8)$ Dirac algebra requires $16 \times 16$ reducible matrices,

$$
\bar{\Gamma}^{I}=\left(\begin{array}{cc}
0 & \Gamma_{A \dot{A}}^{I} \\
\left(\tilde{\Gamma}^{I}\right)_{\dot{B} B} & 0
\end{array}\right)
$$

corresponding to the 16-component Majorana spinors

$$
\Psi=\left(\psi_{s}^{A}, \psi_{c}^{\dot{A}}\right)
$$

which are formed from the Weyl spinors $\psi_{s}^{A}$ and $\psi_{c}^{\dot{A}}$. We denoted $\tilde{\Gamma}^{I}=\left(\Gamma^{I}\right)^{T}$ and the spinorial indices are $A, \dot{A}=1, \cdots, 8$. The $\bar{\Gamma}^{I}$ 's satisfy the algebra

$$
\left\{\bar{\Gamma}^{I}, \bar{\Gamma}^{J}\right\}=2 \delta^{I J}
$$

provided $\Gamma_{A \dot{A}}^{I}$ satisfy the relations

$$
\begin{aligned}
& \Gamma_{A \dot{A}}^{I} \tilde{\Gamma}_{\dot{A} B}^{J}+\Gamma_{A \dot{A}}^{J} \tilde{\Gamma}_{\dot{A} B}^{I}=2 \delta^{I J} \delta_{A B} \\
& \tilde{\Gamma}_{\dot{A} A}^{I} \Gamma_{A \dot{B}}^{J}+\tilde{\Gamma}_{\dot{A} A}^{J} \Gamma_{A \dot{B}}^{I}=2 \delta^{I J} \delta_{\dot{A} \dot{B}} .
\end{aligned}
$$

Similarly, we can also define antisymmetric products

$$
\begin{aligned}
& \Gamma_{A \dot{A}}^{I} \tilde{\Gamma}_{\dot{A} B}^{J}-\Gamma_{A \dot{A}}^{J} \tilde{\Gamma}_{\dot{A} B}^{I}=2 \Gamma_{A B}^{I J} \\
& \tilde{\Gamma}_{\dot{A} A}^{I} \Gamma_{A \dot{B}}^{J}-\tilde{\Gamma}_{\dot{A} A}^{J} \Gamma_{A \dot{B}}^{I}=2 \Gamma_{\dot{A} \dot{B}}^{I J} .
\end{aligned}
$$

The component matrices $\Gamma_{A \dot{A}}^{I}$ can also be treated as real Clebsch-Gordon coefficients. With these $\Gamma$ 's an $S O(8)$ invariant quantity can be constructed by combining the vector and two fermionic representations. We shall be using the real representation

$$
\begin{aligned}
& \Gamma_{1}=\sigma^{2} \times \sigma^{2} \times \sigma^{2}, \\
& \Gamma_{2}=1 \times \tau^{1} \times \sigma^{2}, \\
& \Gamma_{3}=1 \times \tau^{3} \times \sigma^{2},
\end{aligned}
$$




$$
\begin{aligned}
& \Gamma_{4}=\sigma^{2} \times 1 \times \tau^{1} \\
& \Gamma_{5}=\sigma^{2} \times 1 \times \tau^{3} \\
& \Gamma_{6}=\tau^{1} \times \sigma^{2} \times 1 \\
& \Gamma_{7}=\tau^{3} \times \sigma^{2} \times 1 \\
& \Gamma_{8}=1 \times 1 \times 1,
\end{aligned}
$$

where $\sigma^{2}=i \tau^{2}$. See for more details on the representations in [19].

\section{B The topological scalar-tensor 3D action}

Let us discuss here a topological scalar-tensor (ST) action just like we have B-F (Chern-Simons) gauge action in 3D. We can write

$$
S_{S T} \sim \int d^{3} x\left(-\frac{1}{2} \epsilon^{\mu \nu \lambda} C_{\mu \nu}^{I} \partial_{\lambda} \eta^{I}-i \bar{\chi} \zeta\right)
$$

The fermions $\chi^{A}$ and $\zeta^{A}$ make the supersymmetric partners for $\eta^{I}$ and $C_{\mu \nu}^{I}$ and belong to $8_{s}$ representation of $S O(8)$. All the fields in the Lagrangian are nonpropagating (auxiliary) fields. The equations of motion of the fermions are simply

$$
\zeta=0=\bar{\chi}
$$

There is an obvious gauge invariance under $\delta C_{\mu \nu}^{I}=\partial_{[\mu} \lambda_{\nu]}^{I}$.

The action (58) does possess $\mathcal{N}=8$ supersymmetry under the infinitesimal variations

$$
\begin{aligned}
& \delta \eta^{I}=i \bar{\epsilon} \tilde{\Gamma}^{I} \chi, \quad \delta \chi=-\not \partial \eta^{I} \Gamma^{I} \epsilon, \\
& \delta C_{\mu \nu}^{I}=i \bar{\epsilon} \tilde{\Gamma}^{I} \gamma_{\mu \nu} \zeta, \quad \delta \zeta=\frac{1}{2} \gamma^{\mu \nu \lambda} \partial_{\mu} C_{\nu \lambda}^{I} \Gamma^{I} \epsilon .
\end{aligned}
$$

The supersymmetry parameters $\epsilon^{\dot{A}}$ are 8 two-component Majorana spinors belonging to $8_{c}$ representation of $S O(8)$.

The above scalar-tensor action can be constructed in analogy with Chern-Simons gauge action $[20]$

$$
S=-\int d^{3} x\left(\frac{1}{2} \epsilon^{\mu \nu \lambda} A_{\mu} F_{\nu \lambda}+i \bar{\chi} \chi\right)
$$

which is topological in nature. 


\section{Romans' type IIA supergravity: The BdGPT duality}

The massive type IIA maximal supergravity [21] in 10 dimensions is known to have a cosmological constant term proportional to $m^{2}$ alongwith mass terms for 2-rank tensor fields. Due to this the action does not have the known $Z_{2}$ invariance of type II strings, under which RR $p$-form potentials flip their sign, unless the mass parameter simultaneously changes its sign. It was interestingly suggested in [22] that the mass parameter $m$ could, in fact, be lifted to a 0 -form, $F_{(0)}$, which in turn can be Hodge-dualised to a 10-form field strength

$$
F_{(10)} \equiv d A_{(9)}
$$

Particularly, the D8-branes are charged under 9-form potential $A_{(9)}$, which are 1/2BPS solutions of the theory. Under this 'localisation' of the Romans' mass, the action goes over to [22]

$$
L_{m}^{I I A}\left(A_{(p)} ; m\right) \rightarrow L_{m}^{I I A}\left(A_{(p)} ; F_{(0)}\right)+F_{(0)} \wedge d A_{(9)}
$$

In fact, the 9-form potential $A_{(9)}$ plays the role of a Lagrange multiplier field. It imposes the constraint that

$$
d F_{(0)}=0,
$$

i.e. in the vacuum $\left\langle F_{(0)}\right\rangle=m$. The duality relation between $F_{(0)}$ and $F_{(10)}$ is nothing but the $F_{(0)}$ equation of motion

$$
\frac{\delta L_{m}^{I I A}\left(A_{(p)} ; F_{(0)}\right)}{\delta F_{(0)}}=-*_{10} d A_{(9)} .
$$

We call this as Bergshoeff-de-Roo-Green-Papadopoulos-Townsend (BdGPT) duality relation. In this formulation the massive type IIA SUGRA regains the $Z_{2}$ symmetry under which the local fields transform as

$$
F_{(0)} \rightarrow-F_{(0)} \quad \text { and } \quad A_{(9)} \rightarrow-A_{(9)} .
$$

instead of a constant mass parameter $m \rightarrow-m$. It is this similar argument which we incorporated in constructing an $S O(8)$ invariant B-F theory [1]. In that construction

there are eight constant couplings $g^{I}$ which transform under $S O(8)$. However, the theory transforms along with the couplings. So it was needed to make these couplings localised,

$$
g^{I} \mapsto \eta^{I}(x)
$$




\section{References}

[1] H. Singh, Phys. Lett. B673 (2009) 68, arxiv:hep-th/0811.1690.

[2] J. Bagger and N. Lambert, hep-th/0611108; hep-th/0711.0955; hep-th/0712.3738.

[3] A. Gustavsson, arXiv:0709.1260 [hep-th]; A. Gustavsson, JHEP 0804, 083 (2008) [arXiv:0802.3456 [hep-th]].

[4] J Gomis, G. Milanesi and J.G. Russo, arXiv:0804.1012.

[5] J. Gomis, D. Rodriguez-Gomez, M. Van Raamsdonk, H. Verlinde, arXiv:0806.0738v2 [hep-th]; S. Benvenuti, D. Rodriguez-Gomez, E. Tonni and H. Verlinde, arXiv:0805.1087 [hep-th].

[6] M. A. Bandres, A. E. Lipstein and J. H. Schwarz, arXiv:0806.0054 [hep-th]; M. A. Bandres, A. E. Lipstein and J. H. Schwarz, JHEP 0805, 025 (2008) [arXiv:0803.3242 [hep-th]].

[7] O. Aharony, O. Bergman, D. L. Jafferis and J. Maldacena, "N=6 superconformal Chern-Simons-matter theories, M2-branes and their gravity duals," arXiv:0806.1218 [hep-th].

[8] H. Singh, JHEP 0809, 071 (2008) [arXiv:0807.5016 [hep-th]]; C. Krishnan, C. Maccaferri and H. Singh, arXiv:0902.0290 [hep-th].

[9] A. Basu and J.A. Harvey, Nucl. Phys. B 713 (2005) 136 arXiv:0412310 [hep-th].

[10] E. Antonyan and A.A. Tseytlin, arXiv:0811.1540 [hep-th].

[11] S.P. Chowdhury, S. Mukhopadhyay and K. Ray, arXiv:0903.2951 [hep-th].

[12] S. Mukhi and C. Papageorgakis, JHEP 0805, 085 (2008) [arXiv:0803.3218 [hepth]]; J. Distler, S. Mukhi, C. Papageorgakis and M. Van Raamsdonk, JHEP 0805, 038 (2008) [arXiv:0804.1256 [hep-th]].

[13] B. Ezhuthachan, S. Mukhi and C. Papageorgakis, JHEP 0807, 041 (2008) [arXiv:0806.1639 [hep-th]].

[14] I. A. Bandos, K. Lechner, A. Nurmagambetov, P. Pasti, D. P. Sorokin and M. Tonin, Phys. Rev. Lett. 78, 4332 (1997) [arXiv:hep-th/9701149].

[15] P.S. Howe, N.D. Lambert and P.C. West, Nucl. Phys. B515 (1998) 203, hep-th/9709014. 
[16] H. Nicolai and H. Samtleben, Nucl.Phys. B668 (2003) 167-178, [arXiv:0303213 [hep-th]]; B. de Wit, I. Herger, H. Samtleben, Nucl.Phys. B671 (2003) 175216 arXiv:hep-th/0307006; B. de Wit, H. Nicolai, H. Samtleben, arXiv:hepth/0403014.

[17] N.R. Constable, R.C. Myers and O. Tafjord, Phys. Rev. D61 (2000) 106009 [arXiv:9911136 [he-th]].

[18] D. Berenstein, J. Maldacena and H. Nastase, arXiv:hep-th/0202021.

[19] M. Green, J. Schwarz and E. Witten, "Superstring theory", Vol.1, Cambridge (1987).

[20] J.H. Schwarz, JHEP 0411 (2004) 078; arXiv:hep-th/0411077.

[21] L. J. Romans, Phys. Lett. B169 (1986) 374.

[22] E. Bergshoeff, M. de Roo, M. Green, G. Papadopoulos and P. Townsend, Nucl. Phys. B470 (1996) 113, arXiv:hep-th/9601150 . 